\title{
In vitro propagation of an economically important medicinal plant Lawsonia inermis L. through nodal segments
}

\author{
Amit \\ Plant Tissue culture Lab, Department of Botany, Kurukshetra University, Kurukshetra -136119 \\ (Haryana), India \\ Rajkumar \\ All India Jat Hero,s Memorial College, Rohtak, (Haryana), India \\ Narender Singh* \\ Plant Tissue culture Lab, Department of Botany, Kurukshetra University, Kurukshetra -136119 \\ (Haryana), India \\ *Corresponding author. Email: nsheorankukbot11@gmail.com
}

\section{Article Info}

https://doi.org/10.31018/

jans.v13i3.2612

Received: March 8, 2021

Revised: July 27, 2021

Accepted: August 6, 2021

\section{How to Cite}

Amit et al. (2021). In vitro propagation of an economically important medicinal plant Lawsonia inermis L. through nodal segments. Journal of Applied and Natural Science, 13(3), 897 - 906. https://doi.org/10.31018/jans.v13i3.2612

\begin{abstract}
The present investigation aimed to standardize efficient plant regeneration protocol through in vitro culture by using nodal segment for mass multiplication of Lawsonia inermis an economically important medicinal plant species. Mass multiplication of shoots induced on Murashige and Skoog (MS) medium supplemented with different growth regulators like auxins and cytokinins separately and in different combinations. The medium fortified with 6-Benzylaminopurine (BAP) $1.0 \mathrm{mg} / \mathrm{l}+\mathrm{kinetin}(\mathrm{KN}) 1.5 \mathrm{mg} / \mathrm{l}$ explained best compared to all other combinations. In vitro raised plantlets were excised and transferred in half strength MS medium supplemented with different growth regulators like Indole Butyric acid ( IBA) and naphthalene acetic acid (NAA) (0.5$3.0 \mathrm{mg} / \mathrm{l})$ in an experiment that gave rise to rooting. The half strength of MS medium additive with IBA in separate and in different combinations with NAA concentrations (0.5-3.0 mg/l) supported root development. The best response of rooting was obtained on half MS medium fortified with $1.0 \mathrm{mg} / \mathrm{l}$ IBA. The regenerated plantlets were successfully transplanted to pots. Regenerants were transferred to the field conditions and recorded the survival rate.. Among all the carbon sources and gelling agents used, sucrose $(3 \%)$ in combination with 0.8 per cent agar-agar has proved significantly better. Multiple shoots formation with longer shoots were achieved on medium with $1.0 \mathrm{mg} / \mathrm{BAP}$ and $1.5 \mathrm{mg} / \mathrm{Kn}$. Thus, it is possible to develop a large number of plants of $L$. inermis through shoot bud regeneration which can cater for the need of pharmaceutical as well as other industries.
\end{abstract}

Keywords: Auxins, Cytokininis, Gelling agent, Lawsonia inermis, Nodal segment, Thidiazuron

\section{INTRODUCTION}

Lawsonia inermis $\mathrm{L}$. belongs to family Lythraceae commonly known as Heena, Mehndi, Henna (Persian) Mehandi in (Hindi/Urdu) Madayantika , Ranjaka (Ayruvedic) Hinna , Mehndi (Unani) Marithodi, Marudum (Siddha /Tamil). In World, it is distributed in Middle East, Northern Africa, South-west Asia native and along the coast of sea, Mediterranean sea (Hutchison and Dalzial, 1954). In India, it is mainly distributed in Haryana, Rajasthan, Madhya Pradesh and Gujarat. The plant gives rise to heavy white and yellow coloured flowers with a strong scent due to the presence of a highly aromatic compound that are used to prepare perfume from prehistoric time. Moreover, the plant used to dye henna used to dye skin, hair, nails, silk fibre and the leather industry. $L$. inermis is famous for skin and hair dyeing in most parts of the World. Phytochemical screening by using GC-MS analysis of L. inermis leaves revealed that leaves contains different types of compounds like lawsone, tannic acid, mannite , mucilage, gallic acid and 1,4- naphthoquinone (Chaudhary et al., 2010). L. inermis leaves, flowers, seed, stem bark and roots are used in to prevent different diseases likewise rheumatoid arthritis, headache, diarrhea, ulcers, leprosy, fever, leucorrhoea, renal lithias, gastric problems, diabetes mellitus, diabetes insipedus and heart ailments. The bark is used to cure spleen and leprosy jaundice inflation (Sharma et al., 2012). Many prophylactic revealed that heena has been notified as hypoglycemic, immunostimulant, antiinflammatory, hypoglycemic, heptatoprotective, im- 
munostimulant, anti-inflammatory, antifungal and antibacterial agents (Rahmoun et al., 2010, Villemin et al., 2010, Chaibi et al., 2015, Buddhadev and Buddhadev, 2016).

Conventional propagation of this plant is not successful because of certain diseases caused due to environmental impacts that restrict their multiplication rate. High population demography exploits plant resources, particularly medicinal plants, due to resource partitioning resulting in the depletion of plant product quality ( Waman et al., 2019, Moraes et al., 2021 ). Pharmaceutical industries largely depend upon materials procured from naturally occurring, raising concern about possible extinction and providing concern reasons for in vitro propagation of $L$. inermis. In the present investigation, an attempt has been made to develop an effective method for in vitro propagation for large scale production of this plant.

\section{MATERIALS AND METHODS}

For in vitro propagation of $L$. inermis, the MS medium with different concentrations of phytohormone like IAA (0.5-3.0 $\left.\mathrm{mg} \mathrm{l}^{-1}\right)$, a-naphthaleneacetic acid ( 0.5-3.0 mg I $\left.{ }^{-1}\right)$, 6-benzylaminopurine $\left(0.5-3.0 \mathrm{mg} \mathrm{l}^{-1}\right)$, kinetin $(0.5-$ $3.0) \mathrm{mg} \mathrm{l}^{-1}$ ) and sucrose (3\%) were used. The $\mathrm{pH}$ of the medium was regulated 5.8 with the help of $0.1 \mathrm{~N} \mathrm{KOH}$ and $0.1 \mathrm{~N} \mathrm{HCl}$. Different solidifying agents were used, like $8 \%(w / v)$ agar-agar, sago powder, phytagel and gelrite, in different concentrations. Cultures were kept at a particular temperature $25 \pm 2^{\circ} \mathrm{C}$, illuminating light photoperiod of 16 hour light and 8 hour dark. In all the experiments, the chemicals used were analytical grade (Sigma and Aldrich). Routinely, $25 \mathrm{ml}$ of the liquefied medium was poured into culture tubes and $100 \mathrm{ml}$ in flask sealed with cotton plug wrapped with aluminum foil and sterilized in an autoclave at temperature $121^{\circ} \mathrm{C}$ and 15 pounds per square inch pressure for 15 minutes. After three days, the medium was used for inoculation of nodal explants. Nodal explants (1.0$1.5 \mathrm{~cm}$ ) taken from mother plant of $L$. inermis growing in Herbal garden, Department of Botany, Kurukshetra University, Kurukshetra were inoculated on the Murashige and Skoog (1962) medium by using aseptic conditions. The explants were washed with Tween-20 under running tap water to remove dust particles. The explants were then treated with $10 \%$ Sodium hypochlorite solution for 5minutes, then washed with sterilized double distilled water to remove all the traces of sodium hypochlorite under Laminar airflow chamber. After that, the inoculation of nodal explants in MS medium supplemented with different concentrations of growth regulators (0.5-3.0 $\mathrm{mg} \mathrm{l}^{-1}$ ) of cytokinins (BA and $\mathrm{Kn}$ ) and auxins (IAA, NAA, 2, 4-D, IBA and TDZ) alone and in different combinations for shoot induction and proliferation in plant tissue culture tubes were incubated at tempera- ture $25 \pm 5{ }^{\circ} \mathrm{C}$ under $16 \mathrm{~h}$ photoperiod and 8 hours dark with a photosynthetic photon flux density of $40 \mu \mathrm{mol} \mathrm{m}$ ${ }^{-2} \mathrm{~s}^{-1}$. The nodal explants were placed on semi-solid MS media supplemented with different concentrations of different growth regulators of 6-benzylaminopurine, kinetin $\left(0.5-3.0 \mathrm{mg} / \mathrm{l} \mathrm{mg} \mathrm{^{-1 }}\right)$ for bud break and shoot induction. Twenty culture tubes were used for each of the treatments. Observations like number of days required for bud break shoot induction and number of shoots per explant were noted. The culture tubes were regulated by regular sub-culturing at particular intervals of time 25 days on a fresh medium with the same compositions.

The in vitro regenerated plantlets were transferred to full MS and $1 / 2$ MS medium with or without different concentrations of phytohormones (0.5-3.0) $\mathrm{mg} / \mathrm{l}$ of IBA and NAA for roots formation. Growth and proliferation of roots showed that root formation frequency was different in all concentrations of the media. The regenerated plantlet used for root induction effect at half-strength MS medium was found for root initiation and development. The rooted plantlets were separated from the rooting medium and cleaned with double distilled sterile water to separate the agar-agar from the regenerated plantlet. These plantlets were then transferred to pots containing sterile soil: sand: cocopeat: arbuscular mycorrhizal fungi (1:1:1:1). Potted plantlets were covered with transparent plastic polybags to provide high humidity. These were watered at an interval of two days with $1 / 2$ MS strength salts solution for 15 days. Plastic polybags were removed to acclimatize the plantlets under field conditions after one month. Acclimatized plantlets were transferred to pots containing herbal garden soil for maintenance in a greenhouse under normal photoperiod conditions. Recorded viability rate and data were analyzed by using one-way ANOVA and comparison of variance by using a DMRT at $P \leq 0.05$. All statistical tool was using the SPSS software.

\section{RESULTS AND DISCUSSION}

Freshly organized shoots of $L$. inermis were collected from the mature plants. The micropropagated plant can be produced on a large scale for mass propagation, conservation and sustainable utilization. In vitro propagation generally requires a carbon source in the culture medium. Carbohydrates act as a source of energy required for growth, and maintenance cells also act as signalling molecules involved in cellular metabolic control during in vitro studies culture. Among all the different carbon sources used, sucrose has been found to be the best one (Table 1). Similarly, other workers also reported sucrose as the best source of carbon for tissue culture studies(Demo et al., 2008 in Solanum tuberosum, Kang et al., 2018 in Polygonum multiflorum, Zhang et al., 2017 in Moringa oleifera). In most of 
the plants regenerated through micropropagation, sucrose $(2$ to $3 \%)$ was very useful. Sucrose is required to differentiate vascular bundles in tissue cultured plant cells (Aloni, 1980). It also characterizes the major osmotic intrinsic components of the medium and is necessary for a different types of metabolic activities for cell regulations.

The effect of various solidifying agents on in vitro growth was studied using various solidifying agents like agar-agar, gelatin and phytagel. Comparative studies of in vitro propagation on MS medium solidified with different gelling agents and different carbon sources divulge that high bud breaking percentage and survival rate (Table 1 ) were better in medium containing $0.8 \%$ agar -agar and $3 \%$ sucrose compared to other combinations. Similar experimental results were shown by Qrunfleh et al. (2013) in Ficus carica. Gelatin and phytagel were not proved good solidifying agents for $L$. inermis. Generally, many experimental studies have shown that the type of gelling agent used can control tissues' growth under in vitro conditions in bananas and Albizzia lebbeck ( Ramesh and Ramassamy, 2015; Raina, 2017). Therefore, the study on selecting commercial grades of gelling agents is very important.

The method of in vitro propagation is mainly used for plant tissue culture experiments for medicinally as well as economically important plant species. In the present study, MS medium containing various concentrations of BAP, IAA, KN, TDZ individually and their combinations were used. MS medium without growth regulators acted as a control. The explants inoculated on MS medium without growth regulators did not exhibit any shoot induction and multiplication from the nodal meristem even after 40 days of incubation. Therefore, the role of cytokinins in the induction and activation of axillary buds and subsequent proliferation of adventitious shoot buds is well documented (Kumar and Singh, 2007, Binish and Jothi Nayagi, 2019 ). Among different concentrations of BAP used, $1.5 \mathrm{mg} / \mathrm{l}$ showed the best results (Table 2) for shoot induction in comparison to other treatments. In the case of kinetin, medium with $1.5 \mathrm{mg} / \mathrm{KN}$ distributed good shoot induction response. In combinations, MS medium supplemented with BAP $(1.0 \mathrm{mg} / \mathrm{l})+\mathrm{KN}(1.5 \mathrm{mg} / \mathrm{l})$ produced an average number of 18.5 shoots per explants and maximum shoot length, i.e. $6.5 \mathrm{~cm}$ ( Table 3). The maximum number of shoots $(6.13 \pm 0.22)$ were induced from the explants on MS medium supplemented with $2.0 \mathrm{mg} \mathrm{BAP} \mathrm{(} \mathrm{Fig.} 1$ A). The morphogenic response of nodal explants of $L$. inermis was observed in almost all the treatments with cytokinins (BAP and K), as shown in Table 2. MS medium with different concentrations of cytokinins activated the axillary bud, which was present on the nodal segment of the explants. Among all treatments of the two cytokinins tested in this study, BAP was reported more effective compared to kinetin in shoot induction. The frequency of shoot proliferation from the nodal meristem increased with increasing concentration of the

Table 1. Effect of different carbon sources and various solidifying agents on mass multiplication of $L$. inermis.

\begin{tabular}{lllll}
\hline Carbon source & Solidifying agent & Bud break (\%) & $\begin{array}{l}\text { Number of shoots } \\
\text { per explant }\end{array}$ & $\begin{array}{l}\text { Shoot length } \\
\text { (cm) }\end{array}$ \\
\hline Sucrose (3.0\%) & Agar (0.8\%) & $85.6^{\mathrm{a}}$ & $5.00^{\mathrm{ab}}$ & $5.50^{\mathrm{a}}$ \\
Table sugar (3.0\%) & Agar (0.8\%) & $70.0^{\mathrm{d}}$ & $4.25^{\mathrm{e}}$ & $4.30^{\mathrm{d}}$ \\
Sucrose (3.0\%) & Sago powder (15\%) & $73.3^{\mathrm{f}}$ & $3.25^{\mathrm{g}}$ & $3.11^{\mathrm{f}}$ \\
Sucrose (3.0\%) & Sago powder (15\%) & $65.5^{\mathrm{g}}$ & $3.50^{\mathrm{h}}$ & $4.00^{\mathrm{g}}$ \\
Fructose (3.0\%) & Agar (0.8\%) & $65.5^{\mathrm{h}}$ & $3.20^{\mathrm{i}}$ & $5.15^{\mathrm{h}}$ \\
Dextrose (3.0\%) & Agar (0.8\%) & $55.5^{\mathrm{j}}$ & $3.00^{\mathrm{k}}$ & $2.25 \mathrm{j}$ \\
Mannitol (3.0\%) & Agar (0.8\%) & $44.5^{\mathrm{k}}$ & $2.25^{\mathrm{l}}$ & $2.20^{\mathrm{k}}$ \\
Sucrose (3.0\%) & Phytagel (1.0)g/l. & $36.5^{\mathrm{l}}$ & $2.20^{\mathrm{m}}$ & $2.10^{\mathrm{l}}$ \\
Sucrose (3.0\%) & Phytagel(1.5)g/l. & $35.5^{\mathrm{m}}$ & $2.10^{\mathrm{n}}$ & $2.00^{\mathrm{m}}$ \\
Sucrose (3.0\%) & Phytagel(2.0)g/l. & $35.5^{\mathrm{n}}$ & $2.25^{\mathrm{k}}$ & $2.00^{\mathrm{n}}$ \\
Sucrose (3.0\%) & Phytagel(2.5)g/l. & $30.5^{\circ}$ & $2.25^{\mathrm{k}}$ & $2.00^{\mathrm{o}}$ \\
Sucrose (3.0\%) & Gelrite (1.0) g/l. & $30.5^{\mathrm{p}}$ & $2.00^{\mathrm{m}}$ & $1.75^{\mathrm{p}}$ \\
Sucrose (3.0\%) & Gelrite (1.5) g/l. & $28.5^{\mathrm{q}}$ & $2.00^{\mathrm{m}}$ & $1.75^{\mathrm{q}}$ \\
Sucrose (3.0\%) & Gelrite (2.0)g/l. & $25.5^{\mathrm{r}}$ & $1.50^{\mathrm{n}}$ & $1.50^{\mathrm{r}}$ \\
Sucrose (3.0\%) & Gelrite (2.5)g/l. & $25.1^{\mathrm{s}}$ & $1.50^{\circ}$ & $1.25^{\mathrm{s}}$ \\
Sucrose (3.0\%) & Gelrite(3.0) g/l & $20.1^{\mathrm{t}}$ & $1.25^{\mathrm{p}}$ & $1.25^{\mathrm{t}}$ \\
\hline
\end{tabular}

Mean values followed by different letters within a column do not differ significantly at $\mathrm{P} \leq 0.05$ according to Duncan's Multiple Range Test. 
Amit et al. / J. Appl. \& Nat. Sci. 13(3), 897 - 906 (2021)

Table 2. Effect of different concentrations of BAP, KN, IAA and TDZ on shoot induction from nodal segments of $L$. inermis.

\begin{tabular}{|c|c|c|c|c|c|}
\hline $\begin{array}{l}\text { Phytohormone } \\
\text { (mg/l) }\end{array}$ & $\begin{array}{l}\text { Concentrations } \\
\text { ( } \mathrm{mg} / \mathrm{l})\end{array}$ & $\begin{array}{l}\text { Bud break } \\
(\%)\end{array}$ & $\begin{array}{l}\text { No of days } \\
\text { required for } \\
\text { bud break }\end{array}$ & $\begin{array}{l}\text { No of shoots } \\
\text { (Mean } \pm S D)\end{array}$ & 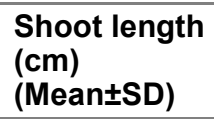 \\
\hline Control & - & - & - & - & - \\
\hline \multirow{6}{*}{ BAP } & 0.5 & 30 & $14.6 \pm 0.11^{\mathrm{d}}$ & $1.50 \pm 0.02^{g}$ & $1.2 \pm 0.23^{9}$ \\
\hline & 1.0 & 70 & $16.5 \pm 0.15^{c}$ & $2.40 \pm 1.13^{\text {ef }}$ & $1.3 \pm 0.33^{\text {ef }}$ \\
\hline & 1.5 & 65 & $18.5 \pm 0.11^{\mathrm{a}}$ & $7.24 \pm 0.04^{a}$ & $2.0 \pm 0.34^{c}$ \\
\hline & 2.0 & 60 & $17.6 \pm 0.15^{b}$ & $4.5 \pm 0.05^{b}$ & $2.4 \pm 0.36^{\mathrm{a}}$ \\
\hline & 2.5 & 65 & $15.5 \pm 0.20^{\mathrm{e}}$ & $3.5 \pm 0.06^{c}$ & $2.5 \pm 0.16^{\mathrm{b}}$ \\
\hline & 3.0 & 60 & $14.2 \pm 0.15^{f}$ & $2.80 \pm 0.05^{\mathrm{d}}$ & $2.6 \pm 0.17^{d}$ \\
\hline \multirow{6}{*}{$\mathrm{KN}$} & 0.5 & 20 & $16.5 \pm 0.55^{\mathrm{b}}$ & $2.4 \pm 0.05^{\mathrm{et}}$ & $2.5 \pm 0.16^{\dagger}$ \\
\hline & 1.0 & 40 & $20.5 \pm 0.45^{\mathrm{a}}$ & $2.5 \pm 0.07^{d}$ & $2.7 \pm 0.17^{\mathrm{a}}$ \\
\hline & 1.5 & 50 & $15.5 \pm 0.35^{\mathrm{c}}$ & $2.7 \pm 0.06^{\mathrm{a}}$ & $2.6 \pm 0.15^{\mathrm{b}}$ \\
\hline & 2.0 & 60 & $12.4 \pm 0.56^{f}$ & $2.6 \pm 0.05^{\mathrm{b}}$ & $2.5 \pm 0.16^{\mathrm{c}}$ \\
\hline & 2.5 & 50 & $15.5 \pm 0.54^{d}$ & $2.5 \pm 0.05^{\mathrm{c}}$ & $2.4 \pm 0.15^{\mathrm{d}}$ \\
\hline & 3.0 & 40 & $13.5 \pm 0.56^{\mathrm{e}}$ & $2.4 \pm 0.06^{\mathrm{g}}$ & $2.5 \pm 0.16^{\mathrm{e}}$ \\
\hline \multirow{6}{*}{ IAA } & 0.5 & 30 & $13.5 \pm 0.05^{\dagger}$ & $5.0 \pm 0.05^{b}$ & $2.6 \pm 0.15^{a}$ \\
\hline & 1.0 & 20 & $25.6 \pm 0.54^{a}$ & $10.5 \pm 0.05^{a}$ & $2.2 \pm 0.16^{\mathrm{c}}$ \\
\hline & 1.5 & 30 & $16.5 \pm 0.56^{b}$ & $4.0 \pm 0.05^{\mathrm{c}}$ & $2.5 \pm 0.16^{\mathrm{b}}$ \\
\hline & 2.0 & 40 & $15.5 \pm 0.14^{c}$ & $3.1 \pm 0.02^{d}$ & $1.5 \pm 0.15^{\mathrm{d}}$ \\
\hline & 2.5 & 30 & $15.0 \pm 0.20^{\mathrm{d}}$ & $1.8 \pm 0.05^{f}$ & $1.5 \pm 0.25^{\mathrm{e}}$ \\
\hline & 3.0 & 35 & $14.0 \pm 0.20^{\mathrm{e}}$ & $2.0 \pm 0.05^{\mathrm{e}}$ & $1.3 \pm 0.15^{f}$ \\
\hline \multirow{6}{*}{ TDZ } & 0.5 & 40 & $16.5 \pm 0.45^{\mathrm{D}}$ & $2.5 \pm 0.05^{\mathrm{a}}$ & $2.6 \pm 0.15^{a}$ \\
\hline & 1.0 & 30 & $15.6 \pm 0.54^{c}$ & $1.5 \pm 0.05^{\mathrm{b}}$ & $2.2 \pm 0.16^{\mathrm{c}}$ \\
\hline & 1.5 & 35 & $22.5 \pm 0.56^{a}$ & $2.0 \pm 0.05^{c}$ & $2.5 \pm 0.16^{b}$ \\
\hline & 2.0 & 55 & $15.5 \pm 0.14^{d}$ & $2.1 \pm 0.02^{d}$ & $1.5 \pm 0.15^{d}$ \\
\hline & 2.5 & 45 & $15.0 \pm 0.20^{f}$ & $2.5 \pm 0.05^{b}$ & $1.5 \pm 0.25^{\mathrm{e}}$ \\
\hline & 3.0 & 55 & $14.0 \pm 0.20^{g}$ & $2.5 \pm 0.05^{\mathrm{b}}$ & $1.3 \pm 0.15^{f}$ \\
\hline
\end{tabular}

Mean values followed by different letters within a column do not differ significantly at $P \leq 0.05$ according to Duncan's Multiple Range Test.

Table 3. Effect of different combination of BAP with $\mathrm{KN}$ on shoot regeneration from nodal explants of $L$.inermis cultured after 25 days of culture.

\begin{tabular}{|c|c|c|c|c|c|}
\hline $\begin{array}{l}\text { Auxins/Cytokinin } \\
\text { (mg/l) }\end{array}$ & $\begin{array}{l}\text { Concentrations } \\
\text { (mg/l) }\end{array}$ & $\begin{array}{l}\text { Bud } \\
\text { break\% }\end{array}$ & $\begin{array}{l}\text { No. of days } \\
\text { required for bud break }\end{array}$ & $\begin{array}{l}\text { No of shoots } \\
\text { (Mean } \pm S D)\end{array}$ & $\begin{array}{l}\text { Shoot length }(\mathrm{cm}) \\
\text { (Mean } \pm \text { SD) }\end{array}$ \\
\hline Control & - & - & - & - & - \\
\hline \multirow{6}{*}{$\mathrm{BAP}+\mathrm{KN}$} & $1.0+0.5$ & 60 & $15.5 \pm 0.45^{c}$ & $15.5 \pm 0.46^{b}$ & $2.1 \pm 0.12^{\mathrm{g}}$ \\
\hline & $1.0+1.0$ & 50 & $16.2 \pm 0.48^{b}$ & $2.4 \pm 0.34^{\mathrm{a}}$ & $5.5 \pm 0.14^{b}$ \\
\hline & $1.0+1.5$ & 65 & $20.5 \pm 0.23^{a}$ & $18.3 \pm 0.43^{f}$ & $6.5 \pm 0.16^{a}$ \\
\hline & $1.0+2.0$ & 70 & $15.1 \pm 0.34^{d}$ & $12.4 \pm 0.45^{d}$ & $4.5 \pm 0.18^{c}$ \\
\hline & $1.0+2.5$ & 60 & $15.00 \pm 0.32^{\mathrm{e}}$ & $10.3 \pm 0.34^{e}$ & $2.5 \pm 0.19^{f}$ \\
\hline & $1.0+3.0$ & 60 & $14.5 \pm 0.12^{f}$ & $14.6 \pm 0.45^{c}$ & $3.5 \pm 0.17^{d}$ \\
\hline \multirow{6}{*}{$\mathrm{BAP}+\mathrm{KN}$} & $1.5+0.5$ & 70 & $12.5 \pm 0.15^{9}$ & $8.5 \pm 0.34^{f}$ & $3.2 \pm 0.14^{\mathrm{e}}$ \\
\hline & $1.5+1.0$ & 60 & $10.5 \pm 0.25^{\mathrm{h}}$ & $7.0 \pm 0.25^{\mathrm{g}}$ & $1.5 \pm 0.24^{\mathrm{h}}$ \\
\hline & $1.5+1.5$ & 65 & $17.6 \pm 0.15^{\mathrm{b}}$ & $2.3 \pm 0.35^{\mathrm{b}}$ & $2.4 \pm 0.25^{\mathrm{a}}$ \\
\hline & $1.5+2.0$ & 65 & $16.5 \pm 0.10^{\mathrm{e}}$ & $2.5 \pm 0.25^{\mathrm{a}}$ & $2.3 \pm 0.26^{c}$ \\
\hline & $1.5+2.5$ & 60 & $15.5 \pm 0.25^{f}$ & $2.3 \pm 0.23^{c}$ & $2.2 \pm 0.24^{d}$ \\
\hline & $1.5+3.0$ & 70 & $14.6 \pm 0.11^{d}$ & $2.0 \pm 0.11^{\mathrm{e}}$ & $1.8 \pm 0.26^{f}$ \\
\hline \multirow{6}{*}{$\mathrm{BAP}+\mathrm{KN}$} & $2.0+0.5$ & 75 & $10.6 \pm 0.45^{b}$ & $2.4 \pm 0.24^{\dagger}$ & $2.4 \pm 0.22^{c}$ \\
\hline & $2.0+1.0$ & 70 & $14.6 \pm 0.25^{\mathrm{e}}$ & $2.5 \pm 0.26^{\mathrm{e}}$ & $2.2 \pm 0.23^{f}$ \\
\hline & $2.0+1.5$ & 60 & $16.5 \pm 0.25^{a}$ & $2.6 \pm 0.25^{\mathrm{b}}$ & $2.2 \pm 0.25^{\mathrm{e}}$ \\
\hline & $2.0+2.0$ & 65 & $15.5 \pm 0.26 c$ & $2.5 \pm 0.24^{d}$ & $2.3 \pm 0.26^{d}$ \\
\hline & $2.0+2.5$ & 60 & $14.5 \pm 0.35^{f}$ & $2.6 \pm 0.27^{\mathrm{a}}$ & $2.4 \pm 0.25^{b}$ \\
\hline & $2.0+3.0$ & 65 & $15.5 \pm 0.25 d$ & $2.5 \pm 0.28^{c}$ & $2.5 \pm 0.26^{a}$ \\
\hline \multirow{6}{*}{$\mathrm{BAP}+\mathrm{KN}$} & $2.5+0.5$ & 65 & $15.5 \pm 0.23^{c}$ & $2.4 \pm 0.24^{\mathrm{e}}$ & $2.5 \pm 0.15^{b}$ \\
\hline & $2.5+1.0$ & 60 & $15.6 \pm 0.21^{b}$ & $2.5 \pm 0.25^{\mathrm{b}}$ & $2.3 \pm 0.13^{\mathrm{e}}$ \\
\hline & $2.5+1.5$ & 70 & $15.2 \pm 0.23^{\mathrm{e}}$ & $2.4 \pm 0.23^{d}$ & $2.4 \pm 0.17^{d}$ \\
\hline & $2.5+2.0$ & 60 & $15.1 \pm 0.24^{f}$ & $2.6 \pm 0.24^{\mathrm{a}}$ & $2.5 \pm 0.17^{a}$ \\
\hline & $2.5+2.5$ & 60 & $15.5 \pm 0.25^{d}$ & $2.5 \pm 0.25^{\mathrm{c}}$ & $2.2 \pm 0.16^{f}$ \\
\hline & $2.5+3.0$ & 70 & $16.5 \pm 0.23^{a}$ & $2.4 \pm 0.26^{f}$ & $2.4 \pm 0.11^{\mathrm{c}}$ \\
\hline
\end{tabular}

Mean values followed by different letters within a column do not differ significantly at $P \leq 0.05$ according to Duncan's Multiple Range Test. 
Table 4. Effect of different combination of TDZ with BAP on shoot regeneration from nodal explants of $L$.inermis cultured after 25 days of culture.

\begin{tabular}{llllll}
\hline $\begin{array}{l}\text { Phytohormone } \\
\text { (mg/l) }\end{array}$ & $\begin{array}{l}\text { Concentrations } \\
\text { ( } \mathbf{m g} / \mathbf{l})\end{array}$ & \%Bud break & $\begin{array}{l}\text { No days required } \\
\text { for bud break }\end{array}$ & $\begin{array}{l}\text { No of shoots } \\
\text { (Mean } \pm \text { SD) }\end{array}$ & $\begin{array}{l}\text { Shoot length } \\
\text { (cm) (Mean } \pm S D)\end{array}$ \\
\hline Control & - & - & - & - & - \\
\hline & $0.5+0.5$ & 45 & $15.6 \pm 0.45^{\mathrm{e}}$ & $3.50 \pm 0.02^{\mathrm{d}}$ & $1.2 \pm 0.23^{\mathrm{g}}$ \\
& $1.0+0.5$ & 60 & $15.5 \pm 0.45^{\mathrm{d}}$ & $5.40 \pm 1.13^{\mathrm{c}}$ & $1.3 \pm 0.33^{\mathrm{ef}}$ \\
& $1.5+0.5$ & 65 & $20.5 \pm 0.55^{\mathrm{b}}$ & $3.24 \pm 0.04^{\mathrm{f}}$ & $2.0 \pm 0.34^{\mathrm{c}}$ \\
& $2.0+0.5$ & 65 & $18.5 \pm 0.66^{\mathrm{c}}$ & $7.5 \pm 0.05^{\mathrm{a}}$ & $2.4 \pm 0.36^{\mathrm{a}}$ \\
& $2.5+0.5$ & 60 & $25.5 \pm 0.55^{\mathrm{a}}$ & $5.5 \pm 0.06^{\mathrm{b}}$ & $2.5 \pm 0.16^{\mathrm{b}}$ \\
& $3.0+0.5$ & 55 & $18.5 \pm 0.45^{\mathrm{f}}$ & $3.80 \pm 0.05^{\mathrm{e}}$ & $2.6 \pm 0.17^{\mathrm{d}}$ \\
& $0.5+1.0$ & 50 & $15.5 \pm 0.11$ & $4.50 \pm 0.05^{\mathrm{a}}$ & $2.5 \pm 0.16^{\mathrm{b}}$ \\
& $1.0+1.0$ & 55 & $14.5 \pm 0.15$ & $3.50 \pm 0.05^{\mathrm{b}}$ & $2.5 \pm 0.16^{\mathrm{b}}$ \\
& $1.5+1.0$ & 50 & $12.5 \pm 0.15$ & $3.60 \pm 0.05^{\mathrm{c}}$ & $2.5 \pm 0.16^{\mathrm{b}}$ \\
& $2.0+1.0$ & 45 & $10.5 \pm 0.15$ & $3.40 \pm 0.05^{\mathrm{d}}$ & $2.5 \pm 0.16^{\mathrm{b}}$ \\
& $2.5+1.0$ & 55 & $12.5 \pm 0.15$ & $2.50 \pm 0.05^{\mathrm{e}}$ & $2.5 \pm 0.16^{\mathrm{b}}$ \\
& $3.0+1.0$ & 55 & $10.5 \pm 0.15$ & $2.40 \pm 0.05^{\mathrm{f}}$ & $2.5 \pm 0.16^{\mathrm{b}}$ \\
\hline
\end{tabular}

Mean values followed by different letters within a column do not differ significantly at $P \leq 0.05$ according to Duncan's Multiple Range Test-

Table 5. Effect of cytokinins in combinations on multiplication of L. inermis.

\begin{tabular}{|c|c|c|c|c|c|}
\hline \multicolumn{2}{|c|}{ Concentrations } & \multirow{2}{*}{$\%$ Bud break } & \multirow{2}{*}{$\begin{array}{l}\text { No. of days required for bud } \\
\text { break (Mean } \pm \text { S.D) }\end{array}$} & \multirow{2}{*}{$\begin{array}{l}\text { No. of shoots } \\
\text { (Mean } \pm S D)\end{array}$} & \multirow{2}{*}{$\begin{array}{l}\text { Shoot length } \\
(\mathrm{cm})(\text { Mean } \pm \text { SD) }\end{array}$} \\
\hline BAP & $\mathbf{K N}$ & & & & \\
\hline 0.5 & 0.5 & 40 & $16.5 \pm 0.45^{\mathrm{c}}$ & $1.0 \pm 0.02^{f}$ & $1.3 \pm 0.33^{f}$ \\
\hline 1.0 & 1.0 & 50 & $15.5+0.35^{d}$ & $1.3 \pm 0.03^{\mathrm{e}}$ & $1.4 \pm 0.22^{\mathrm{e}}$ \\
\hline 1.5 & 1.5 & 60 & $18.5 \pm 0.55^{\mathrm{a}}$ & $2.5 \pm 0.04^{b}$ & $2.0 \pm 0.34^{d}$ \\
\hline 2.0 & 2.0 & 60 & $17.6 \pm 0.66^{b}$ & $2.5 \pm 0.05^{\mathrm{a}}$ & $2.4 \pm 0.36^{c}$ \\
\hline 2.5 & 2.5 & 65 & $12.5 \pm 0.11^{\mathrm{e}}$ & $2.2 \pm 0.06^{d}$ & $2.5 \pm 0.16^{b}$ \\
\hline 3.0 & 3.0 & 60 & $10.5 \pm 0.45^{g}$ & $2.3 \pm 0.05^{\mathrm{c}}$ & $2.6 \pm 0.17^{c}$ \\
\hline 0.5 & 0.5 & 20 & $16.5 \pm 0.55^{c}$ & $2.4 \pm 0.05^{\mathrm{e}}$ & $2.5 \pm 0.16^{b}$ \\
\hline 1.0 & 1.0 & 40 & $17.5 \pm 0.45^{b}$ & $2.0 \pm 0.07^{f}$ & $2.7 \pm 0.17^{a}$ \\
\hline 1.5 & 1.5 & 50 & $18.5 \pm 0.35^{a}$ & $2.5 \pm 0.06^{c}$ & $2.5 \pm 0.15^{\mathrm{d}}$ \\
\hline 2.0 & 2.0 & 60 & $16.4 \pm 0.56^{d}$ & $2.6 \pm 0.05^{b}$ & $2.5 \pm 0.16^{\mathrm{e}}$ \\
\hline 2.5 & 2.5 & 50 & $12.5 \pm 0.54^{f}$ & $2.5 \pm 0.05^{d}$ & $2.4 \pm 0.15^{f}$ \\
\hline 3.0 & 3.0 & 40 & $15.5 \pm 0.56^{\mathrm{e}}$ & $2.7 \pm 0.06^{\mathrm{a}}$ & $2.0 \pm 0.16^{\mathrm{g}}$ \\
\hline
\end{tabular}

Mean values followed by different letters within a column do not differ significantly at $P \leq 0.05$ according to Duncan's Multiple Range Test

cytokinins up to some extent only. The efficacy of BAP over KN during shoot initiation has also been reported by Nabi and Srivastava (2015), Groach and Singh ( 2015). Cytokinins may also produce multiple numbers of shoots from various explants (Agarwal 2015). However, TDZ was best over $\mathrm{Kn}$ and 6-Benzylaminopurine for shoot multiplication in Pogostemon cablin (Moharana et al., 2017). The efficacy of BAP in the context of Kinetin has been described by Murashige (1974) and Zhang et al. (2017). The edge effect of BAP over other cytokinins is well documented in many woody plant species (Kumar and Singh, 2007).

The thidiazuron (TDZ) response individually and in combination could not improve the results (Table 4, 5 and 6 ). The effect of TDZ on in vitro plant regeneration capacity of this species has been reported that prolonged exposure showed harmful effects like passion, exaggeration and even necrosis on growing tips (Faisal et al. 2005, Guo, et al, 2017). Therefore, to devise a systematic and to prevent the negative effects of prolonged exposure of TDZ, an attempt has been made which concentrated on eliminating or minimizing the bad effects of prolonged TDZ exposure and developing an efficient protocol for clonal propagation of $L$. inermis. Supplementation of the auxins and cytokinins in combination ware not proved beneficial for the mass 

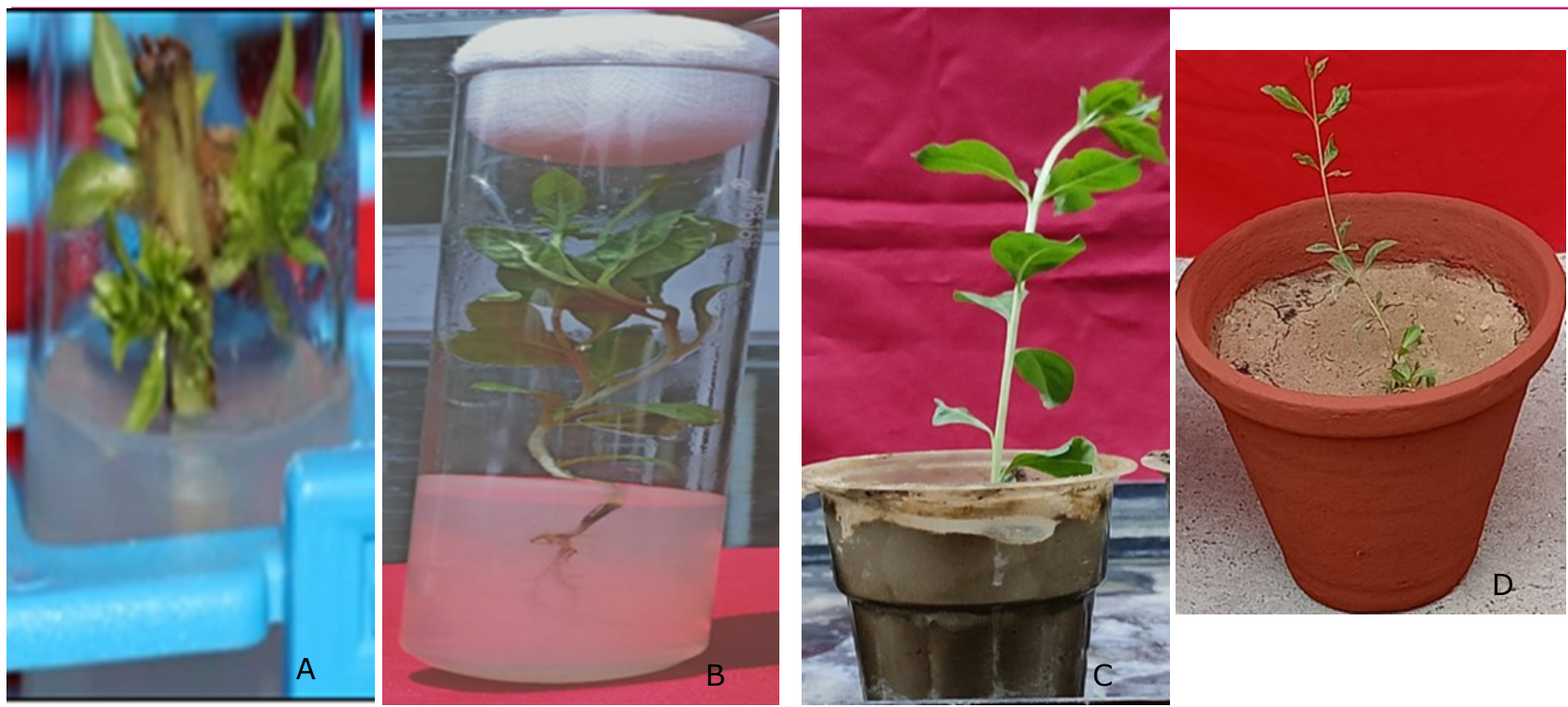

Fig. 1. Regeneration of Lawsonia inermis in full MS medium with different growth regulators: A) Multiple shoot formation on BAP containing MS medium; B) Root formation on $1 / 2$ MS medium NAA and IBA;C) Plantlet established in pot containing sterlized soil, sand, cocopeat and arbuscular mycorrhizal fungi (1:1:1:1) ratio; D) Plantlet transferred to earthern pots under natural conditions after acclimatization.

Table 6. Effect of BAP and KN in combinations with TDZ on nodal segment of $L$. inermis.

\begin{tabular}{|c|c|c|c|c|c|}
\hline (BAP+TDZ)) & Concentrations & $\begin{array}{l}\text { Bud break } \\
(\%)\end{array}$ & $\begin{array}{l}\text { No of days required } \\
\text { for bud break }\end{array}$ & $\begin{array}{l}\text { No of shoots } \\
\text { (Mean } \pm \text { SD) }\end{array}$ & $\begin{array}{l}\text { Shoot length } \\
\text { (cm) (Mean } \pm \text { SD) }\end{array}$ \\
\hline Control & - & - & - & - & - \\
\hline $\mathrm{MS}(\mathrm{BAP}+\mathrm{TDZ})$ & $0.5+0.5$ & 40 & $16.5 \pm 0.45^{c}$ & $1.0 \pm 0.02^{f}$ & $1.3 \pm 0.33^{f}$ \\
\hline $\mathrm{MS}(\mathrm{BAP}+\mathrm{TDZ})$ & $1.0+0.5$ & 50 & $15.5 \pm 0.65^{\mathrm{e}}$ & $1.4 \pm 0.03^{\mathrm{e}}$ & $1.7 \pm 0.11^{\mathrm{e}}$ \\
\hline $\mathrm{MS}(\mathrm{BAP}+\mathrm{TDZ})$ & $1.5+0.5$ & 60 & $20.5 \pm 0.55^{\mathrm{a}}$ & $2.5 \pm 0.04^{b}$ & $2.0 \pm 0.34^{d}$ \\
\hline $\mathrm{MS}(\mathrm{BAP}+\mathrm{TDZ})$ & $2.0+0.5$ & 60 & $17.6 \pm 0.66^{b}$ & $2.5 \pm 0.05^{\mathrm{a}}$ & $2.4 \pm 0.36^{\mathrm{C}}$ \\
\hline $\mathrm{MS}(\mathrm{BAP}+\mathrm{TDZ})$ & $2.5+0.5$ & 65 & $14.5 \pm 0.55^{f}$ & $2.2 \pm 0.06^{d}$ & $2.5 \pm 0.16^{b}$ \\
\hline$M S(B A P+T D Z)$ & $3.0+0.5$ & 60 & $15.5 \pm 0.45^{d}$ & $2.3 \pm 0.05^{c}$ & $2.6 \pm 0.17^{a}$ \\
\hline $\mathrm{MS}(\mathrm{KN}+\mathrm{TDZ})$ & $0.5+0.5$ & 20 & $14.5 \pm 0.55^{\mathrm{g}}$ & $2.4 \pm 0.05^{f}$ & $2.5 \pm 0.16^{\mathrm{e}}$ \\
\hline$M S(K N+T D Z)$ & $1.0+0.5$ & 40 & $13.5 \pm 0.45^{\mathrm{h}}$ & $2.4 \pm 0.07^{\mathrm{e}}$ & $2.7 \pm 0.17^{\mathrm{a}}$ \\
\hline$M S(K N+T D Z$ & $1.5+0.5$ & 50 & $12.5 \pm 0.35^{\mathrm{i}}$ & $2.5 \pm 0.06^{d}$ & $2.6 \pm 0.15^{\mathrm{b}}$ \\
\hline$M S(K N+T D Z$ & $2.0+0.5$ & 60 & $10.4 \pm 0.56^{k}$ & $2.6 \pm 0.05^{b}$ & $2.5 \pm 0.16^{c}$ \\
\hline $\mathrm{MS}(\mathrm{KN}+\mathrm{TDZ}$ & $2.5+0.5$ & 50 & $12.5 \pm 0.54^{j}$ & $2.5 \pm 0.05^{\mathrm{c}}$ & $2.4 \pm 0.15^{\mathrm{e}}$ \\
\hline $\mathrm{MS}(\mathrm{KN}+\mathrm{TDZ})$ & $3.0+0.5$ & 40 & $15.5 \pm 0.56^{\prime}$ & $2.7 \pm 0.06^{\mathrm{a}}$ & $2.5 \pm 0.16^{d}$ \\
\hline
\end{tabular}

Mean values followed by different letters within a column do not differ significantly at $\mathrm{P} \leq 0.05$ according to Duncan's Multiple Range Test

multiplication of this species as these could not improve the bud break and bud proliferation ( Table 5, 6, 7).

The development of roots to the shoots is a necessary step for the sustainability of the plant. Best root formation results were obtained in MS medium supplemented with $0.5 \mathrm{mg} / \mathrm{I}$ IBA ( Table 9, Fig. $1 \mathrm{~B}$ ). MS medium containing $0.5 \mathrm{mg} / \mathrm{l}$ IBA resulted in roots $(75 \%)$ formation within 7 days of inoculation with 7.5 roots per shoot. Among half-strength media, the best results for root formation response was obtained in medium $1 / 2 \mathrm{MS}$ with $0.5 \mathrm{mg} / \mathrm{l}$ NAA ( Table 11). No rooting was recorded in auxin free MS medium ( Table 8, 9, 10 and 11). The promotive effects of auxins on rooting is well established, the nutritive medium has been shown to vary from tissue to tissue as well as species to species ( Kumar and Singh, 2009 in Stevia reboudiana, Lal et al., 2010 in Celastrus paniculatus, Singh et al., 2010 in Sapindus mukorossi, Yu et al., 2017 in Arabidopsis and Oryza sativa, Binish and Jothi Nayagi, 2019 in Ceropegia candelabrum ). The complete regenerated 
Table 7. Effect of auxins and cytokinins supplemented in various combinations on nodal segments of $L$. inermis.

\begin{tabular}{|c|c|c|c|c|c|}
\hline Phytohormone & Concentrations & $\begin{array}{l}\% \text { of bud } \\
\text { break }\end{array}$ & $\begin{array}{l}\text { No. of days } \\
\text { required for }\end{array}$ & $\begin{array}{l}\text { No. of shoots } \\
\text { (Mean } \pm S D)\end{array}$ & $\begin{array}{l}\text { Shoot length } \\
\text { (cm) (Mean士 SD) }\end{array}$ \\
\hline Control & - & - & - & - & - \\
\hline$M S+B A P+I A A$ & $0.5+0.5$ & 40 & $16.5 \pm 0.45^{\mathrm{c}}$ & $1.0 \pm 0.02^{f}$ & $1.3 \pm 0.33^{f}$ \\
\hline$M S+B A P+I A A$ & $1.0+0.5$ & 50 & $15.5+0.35^{d}$ & $1.3 \pm 0.03^{\mathrm{e}}$ & $1.4 \pm 0.22^{\mathrm{e}}$ \\
\hline$M S+B A P+I A A$ & $1.5+0.5$ & 60 & $25.5 \pm 0.55^{a}$ & $2.5 \pm 0.04^{b}$ & $2.0 \pm 0.34^{d}$ \\
\hline$M S+B A P+I A A$ & $2.0+0.5$ & 60 & $17.6 \pm 0.66^{b}$ & $2.7 \pm 0.08^{a}$ & $2.4 \pm 0.36^{c}$ \\
\hline$M S+B A P+I A A$ & $2.5+0.5$ & 65 & $12.5 \pm 0.11^{\mathrm{e}}$ & $2.2 \pm 0.06^{d}$ & $2.5 \pm 0.16^{b}$ \\
\hline$M S+B A P+I A A$ & $3.0+0.5$ & 60 & $10.5 \pm 0.45^{f}$ & $2.3 \pm 0.05^{c}$ & $2.6 \pm 0.17^{\mathrm{a}}$ \\
\hline$M S+K N+I A A$ & $0.5+0.5$ & 20 & $16.5 \pm 0.55^{c}$ & $2.4 \pm 0.05^{\mathrm{e}}$ & $2.5 \pm 0.16^{b}$ \\
\hline$M S+K N+I A A$ & $1.0+0.5$ & 40 & $17.5 \pm 0.45^{b}$ & $2.0 \pm 0.07^{f}$ & $2.7 \pm 0.17^{\mathrm{a}}$ \\
\hline$M S+K N+I A A$ & $1.5+0.5$ & 50 & $18.5 \pm 0.35^{a}$ & $2.5 \pm 0.06^{c}$ & $2.6 \pm 0.15^{\mathrm{c}}$ \\
\hline$M S+K N+I A A$ & $2.0+0.5$ & 60 & $16.4 \pm 0.56^{d}$ & $2.6 \pm 0.05^{b}$ & $2.5 \pm 0.16^{d}$ \\
\hline$M S+K N+I A A$ & $2.5+0.5$ & 50 & $12.5 \pm 0.54^{f}$ & $2.5 \pm 0.05^{d}$ & $2.4 \pm 0.15^{f}$ \\
\hline$M S+K N+I A A$ & $3.0+0.5$ & 40 & $15.5 \pm 0.56^{\mathrm{e}}$ & $2.7 \pm 0.06^{\mathrm{a}}$ & $2.5 \pm 0.16^{\mathrm{e}}$ \\
\hline
\end{tabular}

Mean values followed by different letters within a column do not differ significantly at $P \leq 0.05$ according to Duncan's Multiple Range Test

Table. 8. Root formation on different concentrations of IBA and NAA in L. inermis after 30 days.

\begin{tabular}{|c|c|c|c|}
\hline Media composition (mg/l) & Rooting (\%) & Number of roots & Remarks \\
\hline Control & - & - & - \\
\hline $1 / 2 \mathrm{MS}$ strength $+0.5 \mathrm{IBA}$ & $65.5^{\mathrm{a}}$ & $7.0^{\mathrm{a}}$ & Long and thin \\
\hline $1 / 2 \mathrm{MS}$ strength $+1.0 \mathrm{IBA}$ & $55.5^{\mathrm{c}}$ & $6.5^{\mathrm{b}}$ & Long and thin \\
\hline $1 / 2 \mathrm{MS}$ strength $+1.5 \mathrm{IBA}$ & $45.5^{d}$ & $5.8^{\mathrm{c}}$ & Long and thin \\
\hline $1 / 2 \mathrm{MS}$ strength $+2.0 \mathrm{IBA}$ & $35.5^{\mathrm{e}}$ & $5.0^{\mathrm{d}}$ & Long and thin \\
\hline $1 / 2 \mathrm{MS}$ strength $+2.5 \mathrm{IBA}$ & $30.5^{f}$ & $4.5^{\mathrm{e}}$ & Short and thin \\
\hline $1 / 2 \mathrm{MS}$ strength +3.0 IBA & $25.5^{\mathrm{g}}$ & $4.0^{f}$ & Long and thin \\
\hline $1 / 2 \mathrm{MS}$ strength $+0.5 \mathrm{IBA}+0.5 \mathrm{NAA}$ & $20.5^{\mathrm{h}}$ & $3.5^{\mathrm{g}}$ & Long and thin \\
\hline 1/2MS strength +1.0 IBA +1.0 NAA & $20.5^{\mathrm{i}}$ & $3.0^{h}$ & Long and thin \\
\hline $1 / 2 \mathrm{MS}$ strength +1.5IBA+1.5 NAA & $20.2^{j}$ & $2.7^{\mathrm{i}}$ & Long and thin \\
\hline $1 / 2 \mathrm{MS}$ strength $+2.0 \mathrm{IBA}+2.0 \mathrm{NAA}$ & $20.5^{\mathrm{k}}$ & $2.5^{\mathrm{j}}$ & Short and thin \\
\hline $1 / 2 \mathrm{MS}$ strength $+2.5 \mathrm{IBA}+2.5 \mathrm{NAA}$ & $20.5^{1}$ & $2.0^{\mathrm{k}}$ & Long and thin \\
\hline 1/2MS strength +3.0 IBA +3.0 NAA & $18.5^{\mathrm{m}}$ & $1.5^{\prime}$ & Long and thin \\
\hline
\end{tabular}

Mean values followed by different letters within a column do not differ significantly at $P \leq 0.05$ according to Duncan's Multiple Range Test

plantlets were acclimatized and hardened. About eighty per cent of the plantlets survived and were established well under the field conditions ( Fig. $1 \mathrm{C}$ and D). Thus, it is possible to develop a large number of plants under in vitro conditions of $L$. inermis through nodal segments.

\section{Conclusion}

MS medium containing sucrose (3.0 per cent) in addition to 0.8 per cent agar-agar has proved significantly better for bud break of nodal segments in the present 
Table 9. Root formation on different concentrations of IBA and NAA in L. inermis after 30 days.

\begin{tabular}{|c|c|c|c|}
\hline Media composition (mg/l) & Rooting (\%) & Number of roots & Remarks \\
\hline Control & - & - & - \\
\hline MS strength + 0.5 IBA & $75.5^{\mathrm{a}}$ & $7.5^{\mathrm{a}}$ & Long and thin \\
\hline MS strength + 1.0 IBA & $55.5^{b}$ & $5.6^{b}$ & Long and thin \\
\hline MS strength + 1.5 IBA & $45.5^{\mathrm{c}}$ & $4.5^{\mathrm{c}}$ & Long and thin \\
\hline MS strength +2.0 IBA & $35.5^{d}$ & $4.0^{d}$ & Short and thin \\
\hline MS strength +2.5 IBA & $30.5^{\mathrm{e}}$ & $3.5^{\mathrm{e}}$ & Long and thin \\
\hline MS strength +3.0 IBA & $25.5^{f}$ & $3.0^{f}$ & Long and thin \\
\hline MS strength +0.5 IBA+0.5 NAA & $20.5^{\mathrm{g}}$ & $2.5^{\mathrm{g}}$ & Long and thin \\
\hline MS strength +1.0 IBA +1.0 NAA & $15.5^{\mathrm{h}}$ & $2.0^{\mathrm{h}}$ & Long and thin \\
\hline MS strength +1.5IBA +1.5 NAA & $25.2^{i}$ & $2.0^{i}$ & Long and thin \\
\hline MS strength +2.0 IBA +2.0 NAA & $20.5^{\mathrm{j}}$ & $1.7^{\mathrm{k}}$ & Short and thin \\
\hline MS strength +2.5 IBA +2.5 NAA & $25.5^{\mathrm{k}}$ & $1.5^{1}$ & Long and thin \\
\hline MS strength +3.0 IBA +3.0 NAA & $20.5^{1}$ & $1.0^{\mathrm{m}}$ & Long and thin \\
\hline
\end{tabular}

Mean values followed by different letters within a column do not differ significantly at $P \leq 0.05$ according to Duncan's Multiple Range Test

Table 10. Root formation on different concentrations of NAA in L. inermis after 30 days.

\begin{tabular}{llll}
\hline Media composition (mg/l) & Rooting (\%) & Number of roots & Remarks \\
\hline Control & - & - & - \\
MS strength + 0.5 NAA & $65.5^{\mathrm{a}}$ & $7.8^{\mathrm{a}}$ & Long and thin \\
MS strength + 1.0 NAA & $64.5^{\mathrm{b}}$ & $7.6^{\mathrm{b}}$ & Long and thin \\
MS strength + 1.5 NAA & $60.5^{\mathrm{c}}$ & $7.5^{\mathrm{c}}$ & Long and thin \\
MS strength + 2.0 NAA & $50.5^{\mathrm{d}}$ & $6.5^{\mathrm{d}}$ & Long and thin \\
MS strength +2.5 NAA & $45.5^{\mathrm{e}}$ & $5.5^{\mathrm{e}}$ & Long and thin \\
MS strength +3.0 NAA & $35.5^{\mathrm{f}}$ & $4.5^{\mathrm{f}}$ & Long and thin
\end{tabular}

Mean values followed by different letters within a column do not differ significantly at $P \leq 0.05$ according to Duncan's Multiple Range Test Number of roots.

Table 11. Root formation on $1 / 2$ MS supplemented with different concentrations NAA in L. inermis after 30 days.

\begin{tabular}{llll}
\hline Media composition $(\mathbf{m g} / \mathbf{l})$ & Rooting (\%) & Number of roots & Remarks \\
\hline Control & - & - & - \\
$1 / 2 \mathrm{MS}$ strength & $68.6^{\mathrm{b}}$ & $6.3^{\mathrm{b}}$ & Long and thin \\
$1 / 2 \mathrm{MS}$ strength $+0.5 \mathrm{NAA}$ & $70.5^{\mathrm{a}}$ & $7.0^{\mathrm{a}}$ & Long and thin \\
$1 / 2 \mathrm{MS}$ strength $+1.0 \mathrm{NAA}$ & $60.5^{\mathrm{c}}$ & $6.6^{\mathrm{c}}$ & Long and thin \\
$1 / 2 \mathrm{MS}$ strength $+1.5 \mathrm{NAA}$ & $55.5^{\mathrm{d}}$ & $5.5^{\mathrm{d}}$ & Long and thin \\
$1 / 2 \mathrm{MS}$ strength $+2.0 \mathrm{NAA}$ & $50.5 \mathrm{e}$ & $4.5^{\mathrm{e}}$ & Long and thin \\
$1 / 2 \mathrm{MS}$ strength $+2.5 \mathrm{NAA}$ & $45.5^{\mathrm{f}}$ & $4.5^{\mathrm{g}}$ & Long and thin \\
$1 / 2 \mathrm{MS}$ strength $+3.0 \mathrm{NAA}$ & $40.5^{\mathrm{g}}$ & $3.5^{\mathrm{f}}$ & Long and thin \\
\hline
\end{tabular}

Mean values followed by different letters within a column do not differ significantly at $P \leq 0.05$ according to Duncan's Multiple Range Test 
study. Multiple shoots formation with the higher length of shoots were also achieved on MS medium supplemented with $1.0 \mathrm{mg} / \mathrm{l}$ BAP and $1.5 \mathrm{mg} / \mathrm{l} \mathrm{Kn}$. Thus, through these findings it is possible to develop a large number of plants of $L$. inermis through shoot bud regeneration with higher rate of survival in short span of time. Therefore, the present study has developed a reliable and reproducible protocol of this economically important plant species that could be used for mass multiplication of this species to meet the increasing demand of the pharmaceutical industry and the conservation of germplasm

\section{ACKNOWLEDGEMENTS}

The authors are thankful to Kurukshetra University, Kurukshetra, for providing financial assistance in the form of a project granted under RUSA. ( Rashtriya Uchchatar Shiksha Abhiyan by K. U. Kurukshetra ) The authors are also appreciative to Kurukshetra University, Kurukshetra for providing laboratory facilities and other institutional support.

\section{Conflict of interest}

The authors declare that they have no conflict of interest.

\section{REFERENCES}

1. Agarwal, M. (2015). Tissue culture of Momordica charantia L.: a review. J. Plant Sci., 3, 24-32. doi: 10.11648/ j.jps.s. 2015030101.14

2. Aloni, R. (1980). Role of auxin and sucrose in the differentiation of sieve and tracheary elements in plant tissue cultures. Planta, 150, 255-263. https://link.springer.com/ article/10.1007/BF00390835

3. Binish, T. \& Jothi Nayagi, N. (2019). Micropropagation of endangered medicinal plant Ceropegia candelabrum L.. Pharma Innovation, 8(3), 273-277.

4. Buddhadev, S. G. \& Budhadev, S. S. ( 2016). Ayurvedic medicinal plant Lawsonia inermis Linn.: a complete review., Pharma Science Monitor, 7, 240 -247

5. Chaibi, M., Romdhane, A., Ferchichi, J. \& Bouajila, R (2015). Assessment of antioxidant, anti-inflammatory, anti-cholinesterase and cytotoxic activities of Henna (Lawsonia inermis) flowers, Journal of Natural Products, 8, 85-92.

6. Chaudhary, G., Goyal, S. \& Poonia, P. (2010). Lawsonia inermis Linnaeus: A phytopharmacological review. Int $\mathrm{J}$. Pharm. Sci. Drug Res., 2, 91-98

7. Demo, P., Kuria, P., Nyende, A.B.\& Kahangi, E.M. (2008). Table sugar as an alternative low cost medium component for In vitro micro-propagation of Potato (Solanum tuberosum L.). African J. Biotechnol., 7, 25782584.

8. Faisal, M., Ahmad, N. \& Anis, M. (2005). Shoot multiplication in Rawolfia tetraphylla L. using thidiazuron. Plant Cell Tissue Organ Culture, 80, 187-190. https:// link.springer.com/article/10.1007/s11240-004-0567-x
9. Groach, R. \& Singh, N. (2015). In vitro direct regeneration from cotyledonary node of Suaussurea lappa. International J. Agri. Sci and Res, 5, 361-366.

10. Guo, B., He W, Zhao Y, Wu Y, Fu Y, Guo J, Wei YJPC (2017). Changes in endogenous hormones and $\mathrm{H}_{2} \mathrm{O}_{2}$ burst during shoot organogenesis in TDZ-treated Saussurea involucrate explants. Plant Cell Tissue Organ Culture ,128(1), 1-8. DOI 10.1007/s11240-016-1069-3

11. Hutchinson, J. \& Dalziel, J.M. (1954). Flora of West Tropical Africa. Vol. 1, Part 1. Published by Crown Agents for Oversea Governments and Administrations, London, pp-295.

12. Kang, Y., Lee, Y., Choi ,J., Komakech, R., Min, J., Ju, S., Kim, W.S., Youne, C., Kim, G.Y., Moon, C.B. (2018). Maximizing seedling and root tuber production in Polygonum multiflorum for use in ethnomedicine. $S$ Afr $J$ Bot, 119,119-131.

13. Kumar, S. \& Singh, N.( 2007). An efficient in vitro propagation of a medicinally important plant Stevia reboudiana via nodal segments. Eco-Res. J. Bio- Sci. 6 , 5358.

14. Kumar, S. \& Singh, N.( 2009). Micropropagation of Prosopis cineraria (I.) Druce - a multipurpose desert tree. Researcher, 1, 28-32.

15. Lal D., Singh N. \& Yadav K. (2010). In vitro studies on Celastrus paniculatus. J. Trop. Med. Plant,11, 169-174.

16. Moharana, A., Das, A., Subudhi, E., Naik, S.K.,\& Barik, D..P (2017). Assessment of genetic fidelity using random amplified polymorphic DNA and inter simple sequence repeats markers of Lawsonia inermis L. plants regenerated by axillary shoot proliferation. J Crop Sci Biotech $20,405-416$. https://doi.org/10.1007/s12892-0170002-0.

17. Moraes, R.M.; Cerdeira, A.L. \& Lourenço, M.V. ( 2021). Using micropropagation to develop medicinal plants into crops. Molecules, 26, 1752. https://doi.org/10.3390/mole cules26061752

18. Murashige, T. (1974) Plant propagation through tissue cultures. Annual Review of Plant Physiology, 25, 135166. http://dx.doi.org/10.1146/annurev.pp.25.060174.0 01031

19. Murashige, T. \& Skoog, F. (1962). A revised medium for rapid growth and bio assays with tobacco tissue cultures. Physiologia plantarum, 15(3), 473-497. https://doi.o $\mathrm{rg} / 10.1111 / \mathrm{j} .1399-3054.1962 . t b 08052 . x$

20. Nabi, N. G. \& Srivastava, M. (2015). In vitro propagation of Psoralea corylifolia. Indian J. Applied and Pure Biol, $30,201-205$.

21. Qrunfleh, I.M, Shatnawi, M.M., \& Al-Ajlouni, Z.I. (2013). Effect of different concentrations of carbon source, salinity and gelling agent on in vitro growth of fig (Ficus carica L.). African J Biotechnology, $12,936-940$.

22. Rahmoun, M. N., Benabdallah, M., Villemin, D., Boucherit, K., Mostefa- Kara B, Ziani Cherif C, Choukchou-Braham, N. (2010). Antimicrobial screening of the Algerian Lawsonia inermis (henna). Der Pharma Chemica, 2, 320-326.

23. Raina, R.J., 2017. Gelling agents for plant tissue culture media: a comparative study. International Journal Innovative Research in Science and Engineering, 3(1), 324 331.

24. Ramesh, Y. \& Ramassamy, V., (2015). Influence of gelling agents in micropropagation of banana var. Grand 
Naine. International Journal of Current Research in Biosciences and Plant Biology, 2 (5), 174-178.

25. Sharma, J., Gairola, S. , Gaur,R.D., \& Painuli, R.M. (2012). The treatment of jaundice with medicinal plants in indigenous communities of the sub-himalayan region of Uttarakhand, India. Journal of Ethnopharmacology, 143, 262-291. https://doi.org/10.1016/j.jep.2012.06.034

26. Singh, N., Kaur, A \& Yadav, K. (2010). A reliable In vitro protocol for rapid mass propagation of Sapindus mukorossi Gaertn. Nat. Sci., 8, 41-47

27. Villemin, D., Moreau, B.,Elbilali, A., Didi, M.A., Kaid, M. \& Jaffres, P. A. (2010). Green synthesis of poly(amino methyl enephosphonic) acids. Phosphorus, Sulfur, and Silicon and the Related Elements, 185(12), 2511-2519. https://doi.org/10.1080/10426501003724897
28. Waman, A., Smitha G. R. \& Bohra P. A. ( 2019). Review on clonal propagation of medicinal and aromatic plants through stem cuttings for promoting their cultivation and conservation. Curr. Agri. Res., 7(2), 122-138. DOI:10.12 944/CARJ.7.2.01

29. Yu, J., Liu, W, Liu, J., Qin, P. \& Xu, L. (2017) Auxin control of root organogenesis from callus in tissue culture. front. Plant Sci. https://doi.org/10.3389/fpls.20 17.0 1385.

30. Zhang, J. J., Yang, Y.S., Lin, M.F., Li, S.Q, Tang, Y., Chen, H.B. \& Chen, X.Y. (2017). An efficient micropropagation protocol for direct organogenesis from leaf explants of an economically valuable plant, drumstick (Moringa oleifera Lam.). Ind Crops Prod, 103, 59-63. DOI:10.1016/j.indcrop.2017.03.028 